\title{
Metagenomic Next-Generation Sequencing in the Diagnosis of Deep Sternal Wound Infection After Cardiac Transplantation: A Case Report and Literature Review
}

\author{
Jiongbiao Yu, ${ }^{1}$ Rongjie $\mathrm{Wu},{ }^{2,3}$ Bing Xiong, ${ }^{4}$ Zhifeng Huang, ${ }^{4}$ Hanhua $\mathrm{Li}^{4}$ \\ ${ }^{1}$ Department of General Surgery, The First Affiliated Hospital of Clinical Medicine of Guangdong Pharmaceutical University, \\ Guangzhou, China; ${ }^{2}$ Department of Orthopedics, Guangdong Provincial People's Hospital, Guangdong Academy of Medical \\ Sciences, Guangzhou, China; ${ }^{3}$ Shantou University Medical College, Shantou, China; ${ }^{4}$ Department of Burns and Wound Repair \\ Surgery, Guangdong Provincial People’s Hospital, Guangdong Academy of Medical Sciences, Guangzhou, China
}

\section{ABSTRACT}

Purpose: To explore the value of metagenomics next-generation sequencing (mNGS) for deep sternal wound infection (DSWI) diagnosis.

Methods: mNGS was used to diagnose DSWI after cardiac transplantation; DSWI was treated with surgical debridement, wound care, and antibiotic therapy guided by mNGS.

Results: Coinfection of methicillin-resistant Stapbylococcus aureus (MRSA) and cytomegalovirus (CMV) was detected in this patient. The infection was controlled and the wound healed successfully with the specific medicine based on mNGS results for 3 weeks.

Conclusion: mNGS is effective to achieve precise, individualized, and rapid treatment for wound infection.

\section{INTRODUCTION}

The prevalence of deep sternal wound infection (DSWI) after cardiac surgery reportedly ranges from $1 \%$ to $10 \%$ [Landes 2007; Barnea 2016; Cove 2012; Finkelstein 2005], with a high mortality rate of $10 \%$ to $20 \%$, and even up to 47\% [Kimberly 2011; Braxton 2004], especially for DSWI after cardiac transplantation [Bhatt 2020]. In recent years, rapidly developed metagenomics, high-throughput sequencing, and bioinformatics techniques based on metagenomic next-generation sequencing (mNGS) have become accurate and effective approaches for researchers to thoroughly interpret the composition of pathogenic microorganisms, providing a novel method for pathogenic investigation. Pathogen detection of all microorganisms from the tissue sample is possible by mNGS analysis of DNA or RNA. After removing the background gene data of human beings, and according to the established microbial gene database, results are obtained after big data analysis by integrating the type of

Received fuly 27, 2021; accepted October 21, 2021.

Correspondence: Hanhua Li, Guangdong Academy of Medical Sciences, No. 106, Zhongshan 2 Road, Yuexiu District, Guangdong Provincial, China; +8613632451315 (e-mail: judeyylhb@126.com). specimens, the location of sampling, the patient's medical history, blood picture changes, and other factors. The Illumina sequencing platform is often used, which is fast, accurate, and cost-effective.

In this study, we present a case of successful treatment for DSWI after cardiac transplantation based on the mNGS technique, combined with a literature review to explore the value of mNGS for DSWI diagnosis.

\section{CASE}

The study was approved by the institutional board of Guangdong Provincial People's Hospital, Guangdong Academy of Medical Sciences (no. KY-Q-2021-056-01), and informed consent was obtained from this patient.

The patient is a 52-year-old male who received heart transplantation in our hospital owing to severe heart failure. Immunosuppressants including mycophenolate mofetil and tacrolimus were used regularly after the surgery. Ulceration and purulence in the lower part of the anterior chest wall incision were first detected in the second postoperative week and lasted for a year without any signs of healing. To address this problem, the patient was admitted to the burns and wound repair department for wound repair.

Computed tomography and magnetic resonance imaging showed suppuration in the deep portion of the lower sternum; physical and laboratory examinations were normal (Figure 1). Debridement of osteomyelitis and costochondritis in the lower sternum, removal of sternal fixation wire, and negative pressure therapy were performed, and wound tissue was collected for mNGS, pathologic examination, and bacteria culture.

The mNGS result showed Staphylococcus aureus and cytomegalovirus (CMV) infection, with 4533 and 3 reads, respectively. The bacteria culture showed methicillin-resistant S. aureus (MRSA) infection, which was consistent with the mNGS outcome. Based on the results above, vancomycin was administered, and blood was collected the next day for mNGS to determine CMV infection. The result of blood mNGS confirmed CMV infection, and intravenous ganciclovir (250 mg qd) was applied immediately. 
Results of the mNGS examination

\begin{tabular}{lcccc}
\hline Collection Time (2020) & Sample Type & Method & Report Time (2020) & Results \\
\hline August 12 & Wound tissue & mNGS & August 14 & S. aureus \\
& & & & CMV \\
August 12 & Wound tissue & Bacteria culture & August 17 & MRSA \\
August 14 & Blood & mNGS & August 16 & CMV \\
September 4 & Blood & mNGS & September 6 & Negative \\
\hline
\end{tabular}

CMV, cytomegalovirus; mNGS, metagenomics next-generation sequencing.
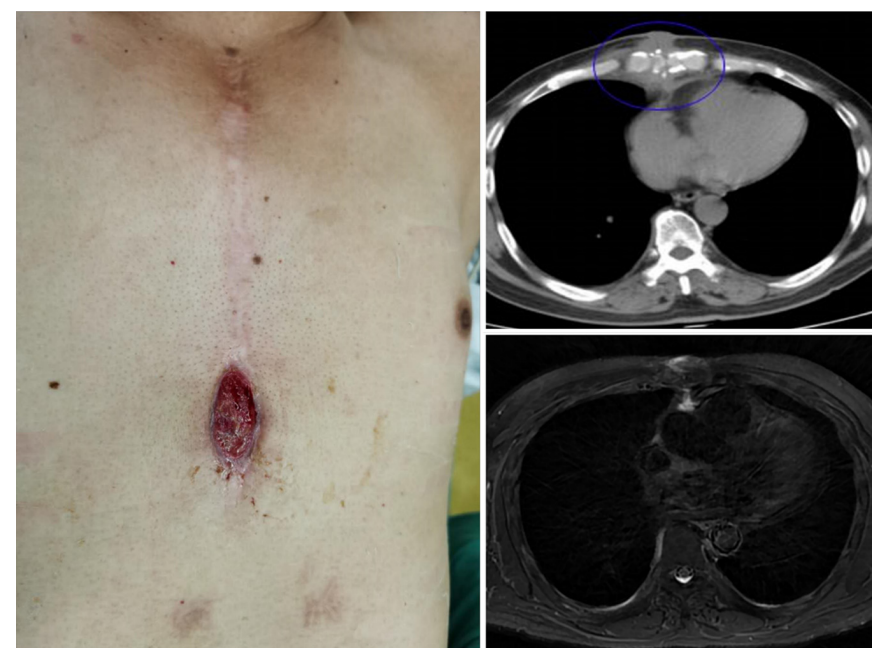

Figure 1. Granulomatous hyperplasia of chest wound with sternal osteomyelitis.

Further debridement of the wound, with reconstruction using muscle flaps from the greater pectoral, was conducted to promote wound healing. The operation was successful, and the patient was discharged after 2 weeks with ganciclovir orally (1000 mg tid). A week after discharge, the patient returned for mNGS re-examination, and the results of the blood sample showed negative cytomegalovirus infection. All mNGS and culture results are shown in Table. After 3 months' follow-up, the patient recovered well, and the wound did not recur (Figure 2).

\section{DISCUSSION}

DSWI is widely accepted as a high-risk postoperative complication of cardiac transplantation that may lead to septic shock or vessel rupture with massive hemorrhage from suture-line damage of the heart. Additionally, immunosuppressants applied postoperatively may promote the spread of infection, which leads to more serious complications [Bhatt 2020]. Thus, early diagnosis with appropriate therapy for DSWI plays an important role in management of infection and prevention of further complications after cardiac surgery.

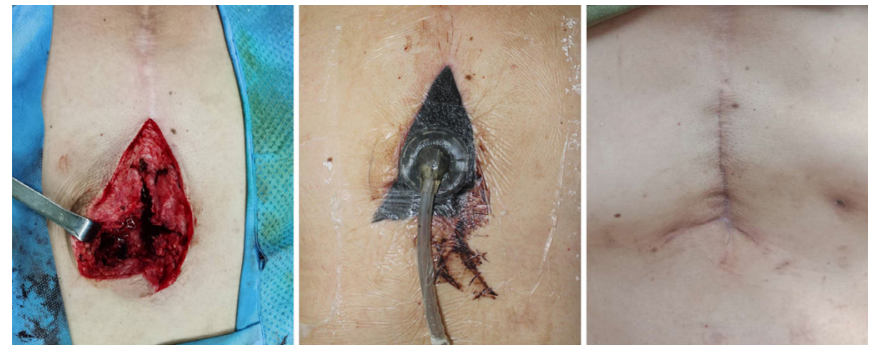

Figure 2. The importance of debridement and negative pressure therapy.

mNGS, a novel technique with advantages of rapid detection, high accuracy, and wide coverage, is regarded as a promising candidate to guide the treatment of deep sternal infection after cardiac surgery.

Generally, patients after cardiac transplantation have a much higher infection rate than healthy people, because immunosuppressants restrain not only immune rejection but also immune ability against pathogens. CMV is the most common pathogen in infection. CMV pneumonia, a common infective complication after organ transplantation, is one of the leading causes of death for immunosuppressive recipients [Razonable 2020]. CMV not only destroys human organs directly, but it also can increase the risk of coinfection with other pathogens such as bacteria and fungi [Walker 1995]. Thus, early detection and treatment of CMV infection are critical for patients after heart transplantation. MRSA colonization, as another serious infective complication in patients undergoing solid organ transplantation, also may be harder to treat with application of immunosuppressants and coinfection with CMV [Ziakas 2014].

Traditionally, pathologic examination plays an important role in the diagnosis and management of infective disease, whereas bacterial culture is the common way to confirm pathogens. In the present study, mNGS took only 2 days to detect $S$. aureus infection, whereas bacterial culture needed 5 days, which was consistent with mNGS results. Although bacterial culture is useful to access antibiotic susceptivity for reasonable antibiotics strategies, its long turnaround time and unavailability for virus detection limit its use.

To overcome the weakness of bacterial culture, mNGS, an advanced diagnostic method, can be used as a superior alternative approach to detect pathogens more rapidly, accurately, 
and comprehensively. In addition to common bacteria, mNGS also detects viruses, anaerobes, and Mycobacterium tuberculosis, providing comprehensive results of the wound infection. Furthermore, as more bacteria drug-resistance gene information is added to the database, mNGS will be a more rapid and accurate method to obtain drug-resistance information and guide antibiotic prescription in comparison with bacteria culture. The examination technique combining bacteriology and molecular biology is regarded as one of the most important advances in medicine; therefore, we believe that in the future, mNGS will facilitate the development of clinical microbiology to achieve precision medicine.

\section{ACKNOWLEDGMENTS}

This research was funded by Administration of Traditional Chinese Medicine of Guangdong Province of China (grant no. 20211012).

\section{REFERENCES}

Barnea Y, Lerner A, Aizic A, et al. Efficacy of dalbavancin in the treatment of MRSA rat sternal osteomyelitis with mediastinitis. Antimicrob Chemother 2016;71:460-463.
Bhatt PJ, Ali M, Rana M, et al. Infections due to multidrug-resistant organisms following heart transplantation: Epidemiology, microbiology, and outcomes. Transpl Infect Dis 2020;22:e13215.

Braxton M, Marrin CA, McGrath PD, et al. 10-year follow-up of patients with and without mediastinitis. Semin Thor Cardiovasc Surg 2004;16:70-76.

Cove ME, Spelman DW, MacLaren G. Infectious complications of cardiac surgery: A clinical review. Cardiothor Vasc Anesth 2012;26:1094-1100

Finkelstein R, Rabino G, Mashiah T, et al. Surgical site infection rates following cardiac surgery: The impact of a 6-year infection control program. Am J Infect Control 2005;33:450-454

Kimberly S, Anderson E, Harper JG. Overview and management of sternal wound infection. Semin Plast Surg 2011;25:25-33.

Landes G, Harris PG, Sampalis JS, et al. Outcomes in the management of sternal dehiscence by plastic surgery: A ten-year review in one university center. Ann Plast Surg 2007;59:659-666.

Razonable RR. Cytomegalovirus in solid organ transplant recipients: Clinical updates, challenges and future directions. Curr Pharm Des 2020;26:3497-3506

Walker RC, Marshall WF, Strickler JG, et al. Pretransplantation assessment of the risk of lymphoproliferative disorder. Clin Infect Dis 1995;20:1346-1353.

Ziakas PD, Pliakos EE, Zervou FN, et al. MRSA and VRE colonization in solid organ transplantation: A meta-analysis of published studies. Am J Transplant 2014;14:1887-1894. 